\title{
EIGENVALUE INTERLACING FOR FIRST ORDER DIFFERENTIAL SYSTEMS WITH PERIODIC $2 \times 2$ MATRIX POTENTIALS AND QUASI-PERIODIC BOUNDARY CONDITIONS
}

\author{
Sonja Currie, Thomas T. Roth And Bruce A. Watson
}

Abstract. The self-adjoint first order system, $J Y^{\prime}+Q Y=\lambda Y$, with locally integrable, real, symmetric, $\pi$-periodic, $2 \times 2$ matrix potential $Q$ is considered, where $J=\left(\begin{array}{cc}0 & 1 \\ -1 & 0\end{array}\right)$. By means of a unitary transformation applied to the boundary value problem considered in [6], it is shown that all eigenvalues to the above equation with boundary conditions $Y(\pi)= \pm R(\theta) Y(0)$, where $R(\theta)$ is the rotation matrix $\left(\begin{array}{cc}\cos \theta & \sin \theta \\ -\sin \theta & \cos \theta\end{array}\right)$, occur when the discriminant $\Delta_{\theta}=\operatorname{Tr}\left(\mathbb{Y}(\pi)^{T} R(\theta)\right)$ is equal to \pm 2 . Here $\mathbb{Y}$ is the solution of the first order system obeying the initial condition $\mathbb{Y}(0)=\mathbb{I}$. In addition, an expression for the $\lambda$-derivative of the discriminant $\Delta_{\theta}$ is given and some monotonicity results are obtained. Interlacing/indexing properties for the eigenvalues of various operator eigenvalue problems are proved.

Mathematics subject classification (2010): 34L40, 34C10, 34C25.

Keywords and phrases: Dirac system, quasi-periodic eigenvalue problems, interlacing.

\section{REFERENCES}

[1] J. Bellissard, B. Iochum, E. Scoppola, D. Testard, Spectral properties of one dimensional quasi-crystals, Commun. Math. Phys. 125 (1989), 527-543.

[2] I. Binder, M. Voda, On Optimal Separation of Eigenvalues for a Quasiperiodic Jacobi Matrix, Commun. Math. Phys. 325 (2014), 1063-1106.

[3] P. A. Binding, H. VolKmer, Existence and asymptotics of eigenvalues of indefinite systems of Sturm-Liouville and Dirac type, J. Diff. Eq. 172 (2001) 116-133.

[4] M. B. Brown, M. S. P. Eastham, K. M. Schmidt, Periodic Differential Operators, Birkhäuser, 2013.

[5] E. A. Coddington, N. Levinson, Theory of ordinary differential equations, McGraw-Hill Publishing, 1955.

[6] S. Currie, B. A. Watson, T. T. Roth, First order systems in $\mathbb{C}^{2}$ on $\mathbb{R}$ with periodic matrix potentials and vanishing instability intervals, Math. Meth. Appl. Sci. 38 (2015), 4435-4447.

[7] L. H. Eliasson, Discrete one-dimensional quasi-periodic Schrödinger operators with pure point spectrum, Acta. Math. 179 (1997), 153-196.

[8] S. G. Krein, Functional Analysis, Nauka, Moskow, 1972.

[9] B. M. Levitan, I. S. SARgSJAn, Sturm-Liouville and Dirac operators, Kluwer Academic Publishers, 1991.

[10] E. J. McShane, Integration, Princeton University Press, 1944.

[11] T. V. Misyura, Characterization of the spectra of the periodic and antiperiodic boundary value problems that are generated by the Dirac operator I, Teor. Funktsii Funktsional. Anal. i Prolozhen., 30 (1978), 90-101.

[12] T. V. Misyura, Characterization of the spectra of the periodic and antiperiodic boundary value problems that are generated by the Dirac operator II, Teor. Funktsii Funktsional. Anal. i Prolozhen., 31 (1979), 102-109. 
[13] I. M. NABIEv, Solution of the Inverse Quasiperiodic Problem for the Dirac System, Matematicheskie Zametki 89 (2011), 885-893.

[14] A. SÜTö, The spectrum of a quasiperiodic Schrödinger operator, Commun. Math. Phys. 111 (1987), 409-415.

[15] J. Weidmann, Spectral theory of ordinary differential operators, Lecture notes in Mathematics 1258, Springer-Verlag, 1987.

[16] C.-F. YANG, X.-P. YANG, Some Ambarzumyan-type theorems for Dirac operators, Inverse Problems, 23 (2007), 2565-2574. 